\title{
Idiopathic spinal cord herniation
}

Abhishek Prasad ${ }^{1}, \mathrm{MD}$, Rahat Brar ${ }^{1}, \mathrm{MD}, \mathrm{DNB}$, Shradha Sinha ${ }^{2}$, DA, DNB, Shaleen Rana ${ }^{1}$, DMRD

ABSTRACT Idiopathic spinal cord herniation (ISCH) is a rare cause of progressive myelopathy. This condition has recently seen an increased frequency of diagnosis, possibly due to increased awareness and the use of magnetic resonance (MR) imaging. ISCH is characterised by herniation of the thoracic spinal cord through an anterior or anterolateral dural defect. Patients usually present with a Brown-Séquard-like syndrome, which is gradually progressive and may evolve into severe paraparesis. This disease has a characteristic radiological appearance, and in most cases, excellent postsurgical outcome. We report ISCH and its imaging appearance in a 31-year-old woman with classical presentation, and discuss the current concepts regarding the aetiopathogenesis, radiological features and management of the disease.

Keywords: dorsal spine, idiopathic spinal cord herniation, $M R$

Singapore Med J 2013; 54(2): e43-e45

\section{INTRODUCTION}

Idiopathic spinal cord herniation (ISCH) is a rare, and most probably, underdiagnosed disorder that is now receiving more attention with the increasing availability of magnetic resonance (MR) imaging. ${ }^{(1)}$ A recent review of the literature found 129 reported cases of $\mathrm{ISCH} .{ }^{(1)}$ This disease is typically diagnosed by its characteristic imaging appearance. Recovery of most of the patient's neurological functions and prevention of the progression of neurological deficits can be achieved with surgical reduction of the herniated spinal cord.(2) However, as not many radiologists or physicians are familiar with the disease, both missed diagnosis and misdiagnosis are common. ${ }^{(3)}$

\section{CASE REPORT}

A 31-year-old Asian woman presented with progressive weakness of the right leg over a period of three years. There was no significant history of trauma, surgery or tuberculosis. Neurological examination revealed spastic asymmetric paraparesis of the right leg, with impaired sense of temperature and pain below the level of T7 thoracic dermatome on the left side. The rest of the general and neurological examinations revealed no significant abnormality. Her laboratory tests were all within normal limits.

The patient underwent MR imaging of the thoracic spine on a 1.5T scanner. Sagittal T2-weighted MR imaging showed focal anterior displacement of the cord at the T6-T7 level, with prominence of the posterior subarachnoid space at this level (Fig. 1). Coronal myelogram (Fig. 2) and axial T1-weighted MR imaging (Fig. 3) at the T6-T7 level revealed herniation of the cord into the right anterolateral aspect through a dural defect, with focal distortion of the cord. T2-weighted axial MR image at the T6 level suggested an associated ventral epidural cyst (Fig. 4). Based on the patient's history and neurological examination, an

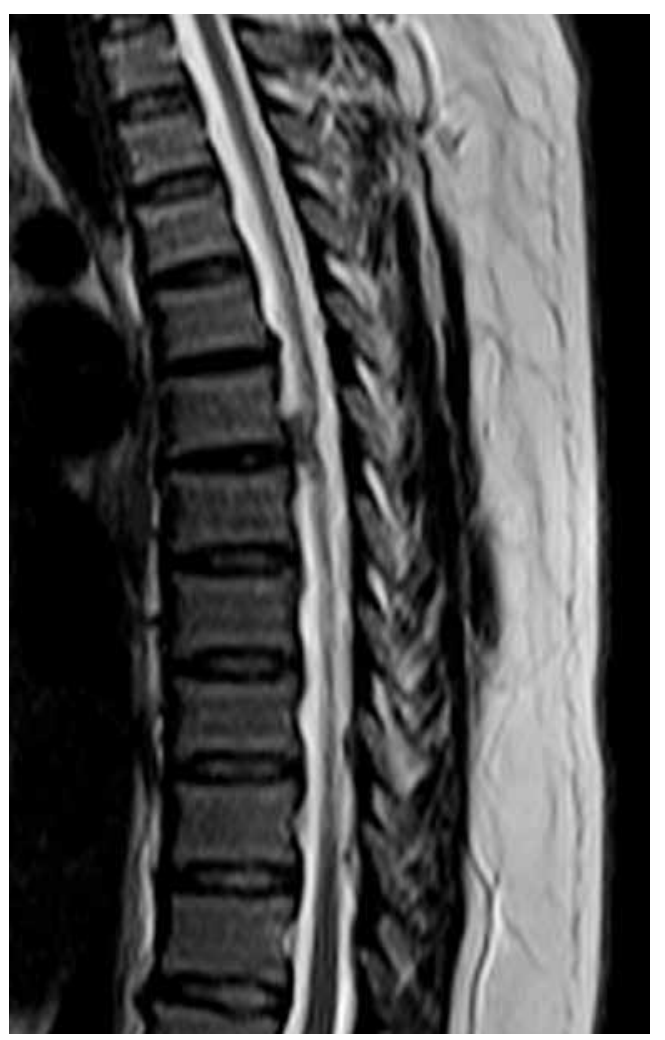

Fig. 1 Midsagittal T2-W MR image of the dorsal spine shows focal anterior displacement of the cord at the T6-T7 level, with prominence of the posterior subarachnoid space at this level.

initial clinical diagnosis of Brown-Séquard syndrome was made. After evaluating the MR imaging results, a diagnosis of ISCH was made.

Posterior laminoplasty and intradural exploration revealed an anteriorly displaced cord herniating through a ventral dural fold, along with the presence of an epidural cerebrospinal fluid (CSF) cyst. The herniated part of the spinal cord was reduced out of the dural defect, and the defect was repaired with a pericardial

${ }^{1}$ Department of Radiology, Fortis Hospital, Mohali, ${ }^{2}$ Department of Anaesthesia, Government Medical College, Chandigarh, India

Correspondence: Dr Abhishek Prasad, Consultant Radiologist, Department of Radiology, Fortis Hospital, Sector 62, Phase VIII, Mohali, Punjab 160062, India. drabhishekprasad@gmail.com 


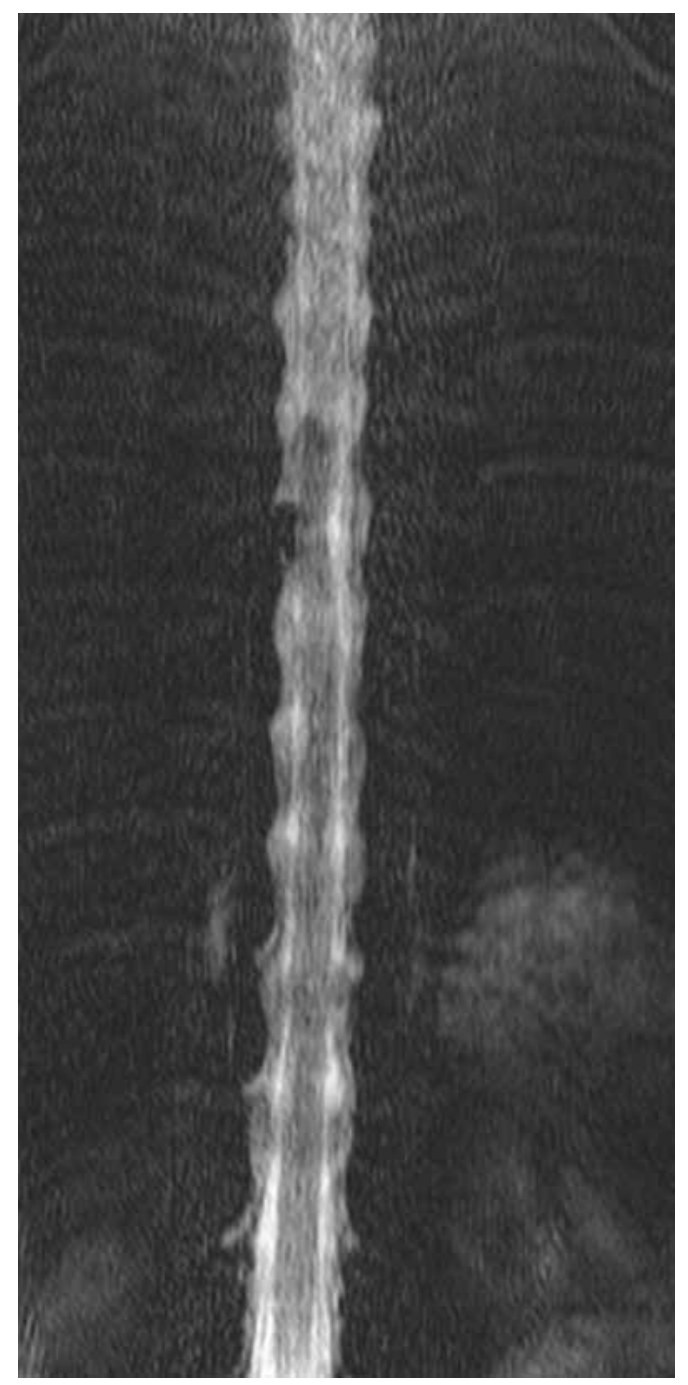

Fig. 2 Coronal myelogram of the dorsal spine shows an extramedullary lesion, which represents the herniated cord. There is slight rightward displacement of the mid-dorsal cord at this level.

graft sling. The patient's weakness improved postoperatively and her sensation returned to normal.

\section{DISCUSSION}

$\mathrm{ISCH}$ is characterised by herniation of the spinal cord through an anterior or anterolateral dural defect. The presence of a dural defect is considered a prerequisite for the development of this condition. CSF enters the extradural space through this defect and forms a localised fluid collection, referred to by some as an extradural arachnoid cyst. Prolonged CSF pulsations can force the cord (especially the closely applied thoracic cord) through the defect. Over time, this leads to adhesions, distortion and possible vascular compromise of the cord, leading to progressive myelopathy. ${ }^{(2)}$

Various causes have been postulated to explain the occurrence of this dural defect ${ }^{(4,5)}$ such as trauma, pressure erosion, thoracic disc extrusion, congenital disorder (pre-existing ventral meningocele), duplication of the ventral dura mater, congenital extradural arachnoid cyst, inflammatory process and congenital abnormal adhesions of the spinal cord to the anterior dura.

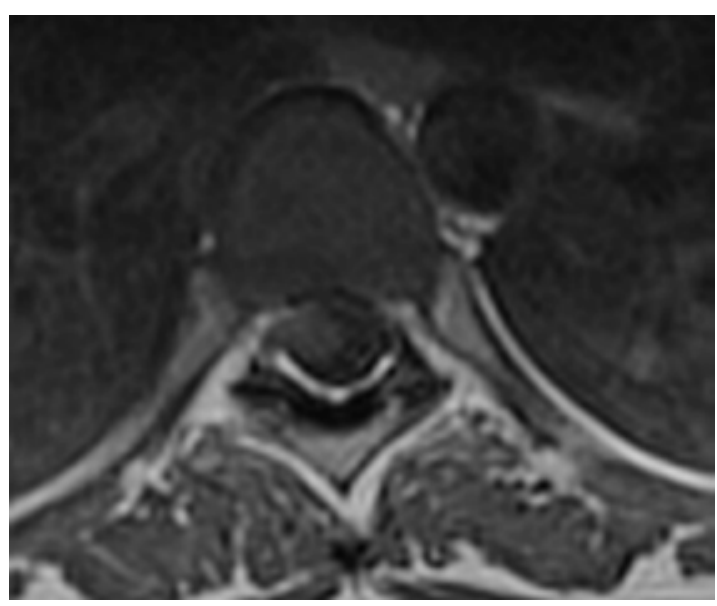

Fig. 3 T1-W axial MR image at the T6-T7 level shows focal herniation of the cord into the epidural space in the right anterolateral aspect, through a dural defect with focal distortion of the cord.

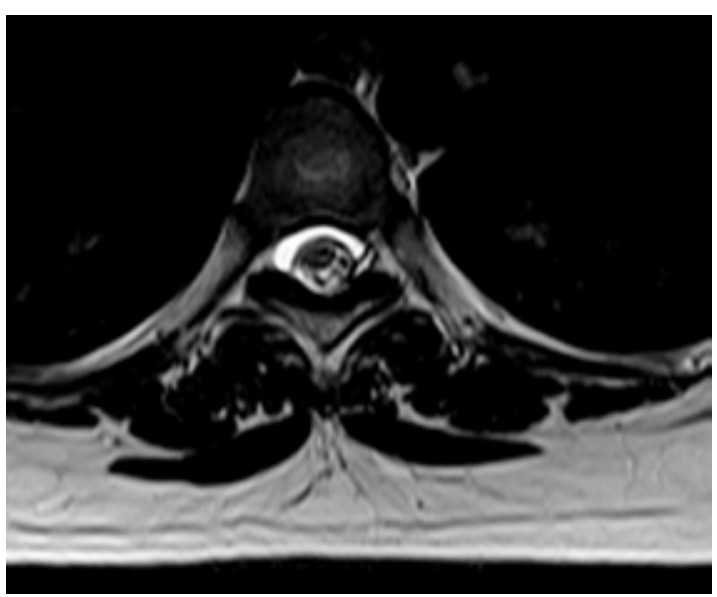

Fig. 4 T2-W axial MR image at a the T6 level shows evidence of an associated ventral epidural cyst.

$\mathrm{ISCH}$ is found in middle-aged adults (range 21-78 years; mean 51 years), with a female preponderance (male to female ratio $1: 1.8) .{ }^{(1)}$ The first tract to be affected by herniation is the lateral spinothalamic tract, which results in diminished pain and temperature sensation, and is frequently unilateral and ascending. As the corticospinal tracts become involved, gradual, progressive weakness and spasticity of the leg(s) also occur. The resultant dissociated sensory deficit with asymmetric spastic paraparesis, known as Brown-Séquard syndrome, is the most frequent clinical presentation of spinal cord herniation, which is seen in nearly two-thirds of reported cases. ${ }^{(1)}$ This was also the case in our patient.

Imaging is the mainstay of diagnosis and nearly always sufficient for the diagnosis of ISCH. ${ }^{(3)} \mathrm{ISCH}$ has a predisposition for the T3-T7 vertebral segments and generally extends over 1-3 cm. However, in one patient, the dural defect was reported to extend over three segments (T4-T6). ${ }^{(6)}$ Sagittal MR images typically show an acute, anterior kink of the thoracic spinal cord at the level of the herniation, with enlargement of the dorsal subarachnoid space. ${ }^{(7)}$ Other findings are an extradural mass-like lesion representing the herniated spinal cord, associated cord atrophy, high T2 signal intensity and scalloping of the vertebral body. ${ }^{(3)}$ 


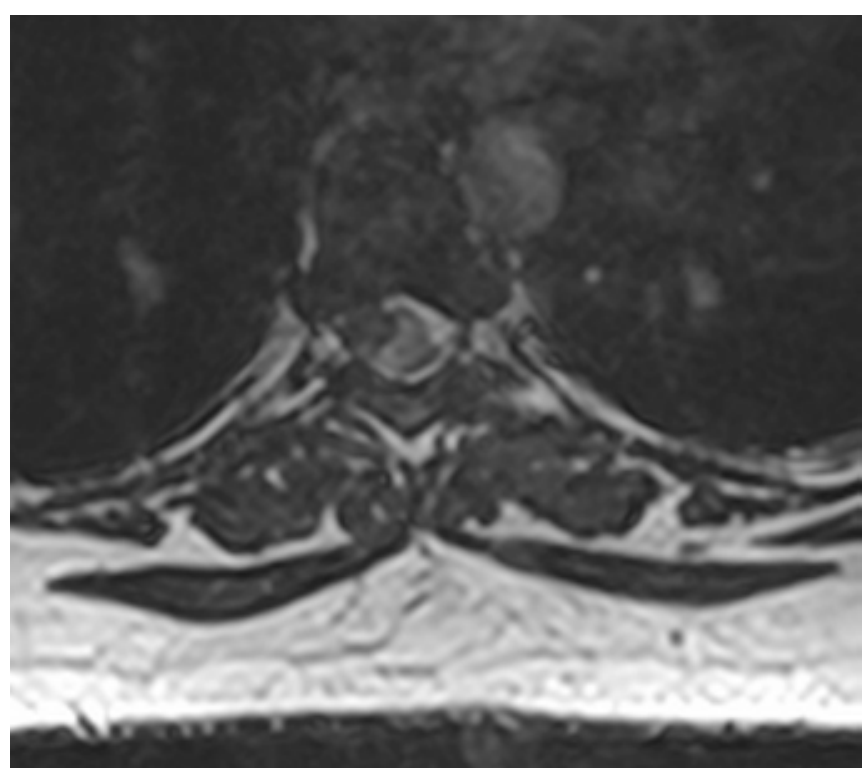

Fig. 5 Constructive interference steady state axial image at the T6-T7 level clearly shows the dural defect and cord herniation.

High-resolution 3D constructive interference in steady state axial and sagittal images have been shown to clearly demonstrate the defect in the thecal sac (Fig. 5). ${ }^{(5)}$ CSF flow in the subarachnoid space posterior to the herniated spinal cord can be seen on phase-contrast pulse cine MR imaging, which helps to exclude the possibility of associated posterior arachnoid cyst. This flow pattern is, however, absent on the ventral side. ${ }^{(7)}$ Conventional thoracic myelography, followed immediately by computed tomography (CT) myelography, ${ }^{(3)}$ shows free movement of myelographic contrast material at the level of the herniated segment. Another imaging feature is the presence of a linear area of hyperattenuation on CT or signal hyperintensity on MR imaging (known as the 'nuclear trail' sign), representing the calcification of nucleus pulposus leakage from a herniated disk. ${ }^{(8)}$ Intradural arachnoid cyst, extradural mass with cord compression and disk herniation with cord tethering are some of the common differential and erroneous diagnoses of $\mathrm{ISCH}^{(6)}$

In a recent study, Imagama et al found that the central type of ventral herniation (as compared to ventrolateral herniation) and associated bone defects have strong relationships with the severity of symptoms and surgical outcome, and are important imaging and clinical features of ISCH. ${ }^{(9)}$ The size of the defect also seems to be important in determining the rate of operative success. In a study by Ishida et $\mathrm{al}^{(10)}$ the cord was found to be herniated through two separate defects of diameters $15 \mathrm{~mm}$ and $5 \mathrm{~mm}$. The cord that herniated into the larger defect could be easily pulled and reduced, whereas the one that herniated into the smaller defect was firm and incarcerated, requiring careful manipulation when detaching from the adherent dura.

The size of the defect has not been mentioned in most case reports on ISCH. Similarly, the size of the defect in our patient had not been noted by the surgeons. However, herniations have almost always been localised to one vertebral segment or a disc, suggesting that the typical length of the defect lies between 1-3 cm. Haussman et al in a report of four cases ${ }^{(11)}$ determined that the length of the defect varied between $0.8 \mathrm{~cm}$ and $3 \mathrm{~cm}$. The width of the defect, however, appears to be less than the diameter of the cord, leading to incarceration of the cord. Dix et $\mathrm{al}^{(2)}$ and Darbar et $\mathrm{al}^{\left({ }^{(6)}\right.}$ reported a defect size of $1.5 \mathrm{~cm} \times 0.6 \mathrm{~cm}$ and $2.00 \mathrm{~cm} \times 0.75 \mathrm{~cm}$, respectively.

$\mathrm{ISCH}$ requires operative treatment in most cases. Operative treatment has been reported to improve motor function in as many as $70 \%$ of patients. The main aims of surgery are to release and realign the spinal cord, and prevent reherniation. This can be achieved either by widening the dural defect, or direct closure or anterior patching of the dural defect. In a meta-analysis of 129 verified operative cases from the literature, ${ }^{(1)}$ a statistically significant association was found among improvement in motor function, widening of the dural defect and subsequent spinal cord release. Brown-Séquard syndrome is also a strong independent factor associated with postoperative motor function improvement, thus emphasising the importance of early identification of ISCH.

In summary, $\mathrm{ISCH}$ is a rare disorder with characteristic MR imaging features. Awareness of the disease is essential for radiologists to achieve early diagnosis, as progression of the patient's neurological deficits can be prevented. Recovery of most neurological functions can be achieved with surgical reduction of the herniated spinal cord.

\section{REFERENCES}

1. Groen RJ, Middel B, Meilof JF, et al. Operative treatment of anterior thoracic Spinal cord herniation: three new cases and an individual patient data meta-analysis of 126 case reports. Neurosurgery 2009; 64(Suppl):145-60.

2. Dix JE, Griffitt W, Yates C, Johnson B. Spontaneous thoracic spinal cord herniation through an anterior dural defect. AJNR Am J Neuroradiol 1998; 19:1345-8.

3. Parmar H, Park P, Brahma B, Gandhi D. Imaging of idiopathic spinal cord herniation. Radiographics 2008; 28:511-8.

4. Blasel S, Hattingen E, Baas H, Zanella F, Weidauer S. Spontaneous Spinal Cord Herniation: MR Imaging and Clinical Features in Six Cases. Clin Neuroradiol 2008; 18:224-30.

5. Najjar MW, Baeesa SS, Lingawi SS. Idiopathic spinal cord herniation: A new theory of pathogenesis. Surg Neurol 2004; 62:161-71.

6. Darbar A, Krishnamurthy S, Holsapple JW, Hodge CJ Jr. Ventral thoracic spinal cord herniation: Frequently misdiagnosed entity. Spine (Phila Pa 1976) 2006; 31:E600-5.

7. Brugieres $\mathrm{P}$, Malapert $\mathrm{D}$, Adle-Biassette $\mathrm{H}$, et al. Idiopathic spinal cord herniation: value of MR phase-contrast imaging. AJNR Am J Neuroradiol 1999; 20:935-9.

8. Spissu A, Peltz MT, Matta G, Cannas A. Traumatic transdural spinal cord herniation and the nuclear trail sign: case report. Neurol Sci 2004; 25:151-3.

9. Imagama S, Matsuyama Y, Sakai Y, et al. Image classification of idiopathic spinal cord herniation based on symptom severity and surgical outcome: a multicenter study. J Neurosurg Spine 2009; 11:310-9.

10. Ishida M, Maeda M, Kasai Y, Uchida A, Takeda K. Idiopathic spinal cord herniation through the inner layer of duplicated anterior dura: evaluation with high-resolution 3D MRI. J Clin Neurosci 2008; 15:933-7.

11. Hausmann ON, Moseley IF. Idiopathic dural herniation of the thoracic spinal cord. Neuroradiology 1996; 38:503-10. 